\title{
REVIEW
}

\section{Gene therapy for liver diseases: recent strategies for treatment of viral hepatitis and liver malignancies}

\author{
V Schmitz, C Qian, J Ruiz, B Sangro, I Melero, G Mazzolini, I Narvaiza, J Prieto
}

Gut 2002;50:130-135

Gene therapy has emerged as a powerful and very plastic tool to regulate biological functions in diseased tissues with application in virtually all medical fields. An increasing number of experimental and clinical studies underline the importance of genes as curative agents in the future. However, intense research is needed to evaluate the potential of gene therapy to improve efficacy and minimise the toxicity of the procedure.

\section{GENERAL ASPECTS}

Gene therapy represents a new and promising therapeutic modality. The underlying principle is based on the introduction of genetic material into cells to generate a curative biological effect. ${ }^{12}$ Gene therapy is not limited to hereditary diseases but can be used for a broad variety of different acquired diseases, such as infections, degenerative disorders, and cancer. The most challenging issues for successful application of gene therapy to human diseases concern: (1) the choice of the relevant therapeutic gene, (2) the choice of promoter and regulatory sequences driving expression of the transgene; and (3) the vector used for delivery of the transgene into cells (that is, for transduction of target cells). Promoter, regulatory elements, and vector characteristics determine transduction efficacy (that is, the number of target cells expressing the transgene and the intensity of gene expression per cell), specificity of the transduction, time of transgene expression, the host's immune response against the vector, and eventually undesired side effects. At present, important efforts are being focused on the search for vectors with less toxicity and prolonged and controlled transgene expression, thereby widening the potential application of gene therapy to a high spectrum of medical fields.

See end of article for authors' affiliations ....................

Correspondence to: Dr Volker Schmitz, Universitätsklinikum Bonn, Medizinische Klinik I, Rhein.

Friedrich-Wilhelms-Universität Sigmund-Freud-Str.25, D-53105 Bonn, Germany; Volker.Schmitz@uni-bonn.de

Accepted for publication 27 June 2001

\begin{abstract}
"In recent years gene therapy has emerged as a new and promising method to treat human diseases"
\end{abstract}

Despite the impressive progress in biomedical sciences during the last decades, the therapy of many liver diseases remains unsatisfactory. This applies not only to primary and metastatic liver tumours, hepatic cirrhosis, and hereditary metabolic diseases but also to a high proportion of cases with chronic viral hepatitis that do not respond to current antiviral therapy. There is evidently an urgent need for efficient alternative therapeutic approaches and in recent years gene therapy has emerged as a new and promising method to treat human diseases.

This article deals with the main features of gene therapy and focuses on recent gene therapeutic strategies for the treatment of viral hepatitis and liver cancer.

\section{Genetic material}

The genetic material used for gene therapy embraces both natural and chimeric genes (which direct the synthesis of therapeutic proteins inside the transduced cells) and also subgenomic DNA and RNA molecules (which act directly to modify expression of endogenous genes). Natural genes are genomic sequences which encode natural proteins such as enzymes, cytokines, costimulatory molecules, antigens, suicide genes, and tumour suppressor genes. Chimeric genes are genomic constructs that do not exist in nature which codify for such molecules as transdominant negative proteins or single chain antibodies.

Subgenomic DNA and RNA sequences include ribozymes, antisense molecules, and RNA decoys, among others. A ribozyme is an RNA molecule that hybridises specifically with a messenger RNA and possesses a catalytic active domain which cleaves the target mRNA. ${ }^{45}$ Antisense molecules are DNA or RNA constructs which bind complementary sequences by hybridisation and interfere with pre-mRNA processing and messenger RNA translation and accelerate mRNA degradation. ${ }^{67}$ RNA decoys are RNA sequences which ligate transactivating proteins as a substitute for their original binding domain thus blocking the function of the regulatory proteins. ${ }^{8}$

\section{Gene therapy vectors}

Both viral and non-viral vectors are used to transfer genetic material to the inside of target cells. In general, non-viral vectors have lower transduction efficiency and allow for shorter duration of transgene expression than viral vectors. The ideal vector should have low antigenic potential, high capacity, high transduction efficiency, it should allow controlled and targeted transgene expression, it should be produced at reasonable expense,

Abbreviations: ITR, inverted terminal repeat; AAV, adeno associated virus; HSV, herpes simplex virus; IFN- $\alpha$, interferon $\alpha$; IL, interleukin; AFP, $\alpha$ fetoprotein; GM-CSF, granulocyte macrophage-colony stimulating factor; $\mathrm{AdCE} 1$, adenovirus containing the core and $E 1$ sequences from hepatitis $C$ virus; AdlL-12, adenovirus containing genes of IL-12; WHV, woodchuck hepatitis virus; HCC, hepatocellular carcinoma; HSV-tk, HSV-thymidine kinase. 
and it has to be safe for both patient and the environment. Gene delivery vehicles must be selected according to the specific therapeutic aim. For some purposes vectors allowing transient gene expression would be adequate while for other medical applications long term expression of the transgene is necessary. In the setting of long term expression, regulation of the activity of the promoter driving the transgene is frequently required. The perfect vector covering all therapeutic and safety requirements does not exist and much work is needed in this important research field. ${ }^{9}$

\section{Non-viral vectors}

Non-viral vectors bear some advantages with respect to viral vectors: handling is easier, capacity for DNA sequences is very high, toxicity is low, they can be specifically targeted to a tissue, and they are not immunogenic thus permitting repeated vector administration.' These advantages however are compounded by a relatively low transduction efficiency. Non-viral vectors include liposomes, DNA-protein complexes, and naked DNA. The latter can be injected into tissues directly or as DNA coated gold particles using the gene gun.

\section{Liposomes}

This gene transfer system consists of DNA surrounded by a liposomal coat that allows adsorption to the cell membrane and penetration inside the cell by endocytosis. The transduction efficiency of this system is determined by liposome size and lipid formulation. ${ }^{10}$ The affinity of liposomes for the target cells can be modified by incorporating monoclonal antibodies into the lipid complexes. These vectors are known as immunoliposomes. ${ }^{11}$ As DNA can be degraded by lysosomes, viral proteins can be incorporated into the liposome to elude the lysosome pathway. These constructs are termed virosomes $^{12}$

\section{Particle mediated bombardment or gene gun}

DNA coated microparticles (tungsten or gold) are delivered into cells by devices using gas pressure as the propelling force. ${ }^{13}$ Penetration of the microbullets is restricted to superficial cell layers of the skin or of the treated organ. ${ }^{14}$

\section{DNA-protein complexes}

Hepatocytes express specific membrane receptors, such as the asialoglycoprotein or the transferrin receptor. These membrane molecules can be used for receptor mediated endocytosis of DNA complexed with the ligand of the receptor. ${ }^{15}$ Transduction efficacy of this system is limited partly by DNA degradation due to lysosomal activity.

\section{Naked DNA}

Plasmid DNA directly injected into tissues has been shown to be incorporated by different cell types, such as muscle, skin cells, and liver cells. ${ }^{16}{ }^{17}$ Despite degradation of DNA within a few days after administration, this technique can be used successfully to generate efficient immune responses against the protein encoded by the injected DNA.

\section{Viral vectors}

Viral vectors are the most efficient vehicles for gene transfer. Different viruses have served to construct gene therapy vectors, including adenovirus, retrovirus, adeno associated virus, herpesvirus, lentivirus, baculovirus, SV40 virus, vacciniavirus, and others.

\section{"Viral vectors are the most efficient vehicles for gene transfer"}

The list of viral vectors is still expanding and modifications of already existing systems will widen the list of potential applications of gene therapy.

\section{Retrovirus}

Murine retroviruses are single stranded RNA viruses which after interaction with a retrovirus receptor can integrate in the genome of a dividing cell. ${ }^{18}$ As hepatocytes do not proliferate actively under physiological conditions, liver tissue is difficult to transduce with retroviral vectors. Furthermore, transduction efficacy is limited by the low titre of virus obtained with the production procedures currently used. As in vitro infection of hepatocytes leads to higher transduction efficacy, retrovirus can however be utilised for ex vivo gene transfer. ${ }^{19}{ }^{20}$ The recent development of human lentiviral (human immunodeficiency virus) based vectors offers promising perspectives for gene transfer into non-dividing cells. ${ }^{21}$

\section{Adenovirus}

These are double stranded DNA viruses with a natural tropism for the liver when administrated systemically, ${ }^{22}$ although liver tropism varies between different species. They can infect very efficiently both dividing and non-dividing cells and are easy to produce at high titres. ${ }^{23}$ Transgenes are expressed transiently because of the host's immune response against viral proteins and the lack of DNA integration into the host's genome. ${ }^{24-26}$ With a first generation adenovirus, transgene expression is limited to a period of about two weeks and repetitive systemic administration of adenovirus is ineffective because of inactivation of the vector by humoral antibodies. In first generation adenoviruses, the El (and E3) region is deleted rendering it replication defective. Combined deletion of $\mathrm{E} 1$ and $\mathrm{E} 3$ results in transgene capacity of up to 8 $\mathrm{kb}$. Second and third generation adenoviruses include changes in the E2 region or deletion of E4, respectively. Recently, the so-called gutless adenoviruses have been developed. These vectors lack all viral sequences except the packaging signals (inverted terminal repeats (ITR)). As a result they have low toxicity, very high capacity, and do not elicit immunological responses against the vector, thus allowing prolonged transgene expression. ${ }^{27}$ Gutless adenoviruses are considered to be very promising tools for a number of medical applications of gene therapy, not only for diseases concerning the liver but also, because of the low toxicity, for disorders affecting the central nervous system. Other research avenues aim to construct tumour specific oncolytic adenoviruses by modifying the ElA and/or ElB regions of first generation adenoviruses. ${ }^{28}$ In this way, conditional replicating adenoviruses can be constructed by placing the El gene of the adenoviral genome under the control of a tumour specific promoter such as AFP promoter. Since AFP expression (which normally occurs in fetal liver) can be reactivated in hepatocellular carcinoma (HCC) cells this would restrict adenovirus replication and the cytopathic effect only to these tumoral cells. ${ }^{29}$

Adeno associated virus (AAV)

These are non-pathogenic human parvoviruses which, after deletion of all viral genes except ITR, have been used with notorious success as gene therapy vectors. Adeno associated virus (AAV) vectors can transduce dividing and non-dividing cells and allow long term transgene expression either by integration into the host genome or by persistence in the cells in an episomal form. ${ }^{30}$ After systemic injection, AAV demonstrates significant liver tropism. It has been shown that AAV mediated gene transfer of factor IX to mouse liver induces persistent curative levels of active factor IX. ${ }^{31}$ Adjuvant treatment with genotoxic agents (such as gamma irradiation) enhance expression of the AAV transgene both in vitro and in vivo. ${ }^{32-34}$ AAV based vectors, as indicated for gutless adenoviruses, have considerable potential in the therapy of central nervous system disorders and also in diseases affecting muscle, a cell type that is readily transduced by this type of vector. 
Herpes simplex virus (HSV)

Herpes simplex virus (HSV) is a promising vector for gene transfer, especially to the nervous system, because of its characteristic neurotropism. Additionally, HSV transduces effectively murine liver tissue in vivo. ${ }^{35}$

\section{GENE THERAPY FOR LIVER DISEASES}

The possibility of transferring functional genes to liver cells creates an entirely new horizon in the therapy of liver diseases that will undoubtedly be explored intensely in the coming years. In this review we will focus on experimental data relating to gene therapy of viral hepatitis and of primary and metastatic liver cancer.

\section{Gene therapy for viral hepatitis B and C}

Less than $40 \%$ of patients with chronic hepatitis B and less than $20-30 \%$ of patients with chronic hepatitis C (results vary according to viral genotype and viral load) can be treated successfully by administration of interferon $\alpha$ (IFN- $\alpha)$. The combination of IFN- $\alpha$ and ribavirin increases the response rate in patients infected with genotype $1^{36}$ but no more than $40 \%$ of patients respond to this therapy. It has recently been shown that pegylated IFN- $\alpha$ generates sustained levels of IFN- $\alpha$ in blood with a weekly dosage of the drug. This pharmacological formulation results in a higher response rate (almost $40 \%$ of cases) than with conventional interferon therapy. ${ }^{37}$ Despite these improvements, a high percentage of patients with chronic hepatitis $\mathrm{C}$ are still resistant to existing antiviral therapies.

Gene transfer of IFN- $\alpha$ to the liver using a long term expression vector could induce prolonged and continuous IFN- $\alpha$ production by the proper diseased liver. One preliminary experimental study has recently demonstrated that administration of an adenovirus expressing IFN- $\alpha$ was effective in the prevention of viral hepatitis in a mouse model. ${ }^{38}$ There is a need for testing the therapeutic efficacy and toxicity of IFN- $\alpha$ gene transfer to the liver using appropriate long term expression vectors in animal models of chronic viral hepatitis (such as in the woodchuck model) .

Antisense DNA/RNA or ribozymes are therapeutic molecules that can also be used to treat viral hepatitis. ${ }^{39}{ }^{40}$ By conveying these molecules in long term expression vectors capable of integration into the host genome, a certain proportion of hepatocytes (and their progeny) would be rendered resistant to viral infection. As these cells may enjoy a biological advantage over infected cells, it is hoped that replacement of dead hepatocytes might take place preferentially by the transduced cells which finally may repopulate the entire liver. These assumptions should be tested in the future in experimental animal models.

Gene therapy can also be used to induce prophylactic or therapeutic antiviral immunity. Injection of naked DNA or of vectors, such as Semliki Forest virus vectors containing viral genes, has been shown to be an efficient system to induce protective antiviral immune responses. ${ }^{41}$ Administration of genes coding for viral antigens can be combined with genes encoding immunostimulating molecules such as interleukin (IL)-2, IL-12, or granulocyte macrophage-colony stimulating factor (GM-CSF). ${ }^{42}$ Recently, we have shown that the combined administration of an adenovirus containing the core and El sequences from hepatitis $C$ virus (AdCEl) and another one containing the genes of IL-12 (AdIL-12) resulted in the generation of a cellular THl immune response which was more intense than that obtained after injection of AdCEl alone. ${ }^{44}{ }^{45}$ In a recent work we found that gene gun bombardment with DNA containing the nucleocapsid of woodchuck hepatitis virus (WHV) induced a TH2 type of response which did not protect against a posterior injection of the virus. However, when a plasmid containing WHV core gene was given together with another plasmid coding for woodchuck IL-12, T cell immunity was of the THI type and was strongly protective against viral inoculation. ${ }^{46}$ Thus combined vaccination with both viral and cytokine genes may be a promising immunisation method allowing the induction of a desired pattern of $\mathrm{T}$ cell immunity.

\section{GENE THERAPY FOR NEOPLASTIC LIVER DISEASES}

Malignant diseases of the liver rank fifth among all primary neoplastic diseases. ${ }^{47}{ }^{48} \mathrm{HCC}$ is the most common primary liver malignancy with a rising incidence worldwide. ${ }^{47-49}$ In addition to primary tumours, the liver is the most common organ where tumour metastases occur. ${ }^{49}{ }^{50}$ Both unresectable HCC and liver metastases of digestive tumours lack effective therapy ${ }^{47} 48$ and new therapeutic modalities are urgently needed.

Transfer of therapeutic genes to the tumour mass or to the peritumoral tissue provides a promising new approach for cancer therapy. ${ }^{52-54}$ In animals, different anticancer gene therapy strategies have shown promise, including (a) transfer of tumour suppressor genes (for example, wild-type p53), (b) antisense strategies aimed at inhibiting oncogene expression, (c) transduction of tumour cells with suicide genes (such as HSV-thymidine kinase (HSV-tk)), to sensitise cells to specific compounds (ganciclovir in the case of HSV-tk), (d) genetic immunotherapy to stimulate the host's antitumoral immune response using immunostimulatory cytokines, chemokines, costimulatory molecules, combination of cytokines and chemokines, or genetic vaccination with tumoral antigens, and (d) transfer of genes which interfere with the biological programme of tumour growth (for example, antiangiogenic substances).

\section{"Transfer of therapeutic genes to the tumour mass or to the peritumoral tissue provides a promising new approach for cancer therapy"}

In the following paragraphs we will concentrate mainly on suicide genes and genetic immunotherapy.

Drug sensitisation (suicide genes)

This therapy is based on the transfer of a gene encoding a foreign enzyme which converts a non-toxic prodrug into a lethal drug compound. HSV-tk is the best characterised suicide gene. Expression of functional enzyme HSV-tk in transduced (tumour) cells induces conversion of the non-toxic prodrug ganciclovir into a toxic phosphorylated compound which terminates DNA synthesis and inhibits DNA polymerase. ${ }^{55} \mathrm{~A}$ characteristic trait of the suicide genes is the so-called bystander effect caused by diffusion of the toxic drug metabolite from the transduced cells to the surrounding tissue thus increasing the number of tumoral cells destroyed by the procedure. Because of this effect, significant tumour regression can be achieved even when only a limited percentage of neoplastic cells has been transduced. ${ }^{56}{ }^{57}$ The bystander effect also derives from necrosis of tumoral tissue which induces local inflammation, attraction of dendritic cells, and stimulation of antitumoral immunity. Thus a synergistic antitumoral effect has been observed between suicide gene based therapy and gene transfer of immunostimulatory molecules. ${ }^{58}$

Several studies have demonstrated the efficacy of the HSV-tk/ganciclovir system for the treatment of HCC $^{56960}$ and metastatic colorectal cancer. ${ }^{6162}$ One of the main obstacles limiting the application of this therapy is related to toxic side effects affecting non-tumoral tissue. ${ }^{60}{ }^{63}$ These side effects can be reduced by injecting the vector locally inside the tumour or by the use of tumour specific promoters (such as AFP promoter for HCC) to limit HSV-tk expression to tumour tissue $^{64}$ 


\section{Genetic immunotherapy}

The host's immunity frequently fails to eliminate malignant tumour tissue either due to the lack of recognisable tumour antigens or to the inability of tumour antigens to stimulate an effective immune response. ${ }^{65}{ }^{66}$ Defective antitumour immunity can be attributed partly to the lack of expression of MHC molecules by tumour cells and also to secretion of immunosuppressive factors (such as transforming growth factor $\beta$ or vascular endothelial growth factor) by the tumour. ${ }^{65}$ Gene transfer of cytokines which are important in the regulation of the immune system can overcome the immune tolerance against tumoral antigens thus facilitating tumour rejection. Different cytokines (IL-2, IL-4, IL-6, IL-7; IL-12, INF- $\gamma$, tumour necrosis factor $\alpha$, GM-CSF) have been used to modulate the host's immune response by either ex vivo or in vivo gene transfer. ${ }^{67-70}$ IL-12 is among the most potent cytokines at stimulating antitumour immunity. IL-12 acts by (a) induction of a TH1 type of response, (b) activation of natural killer cells and cytotoxic $\mathrm{T}$ lymphocytes, (c) enhanced expression of adhesion molecules on endothelial cells thus facilitating the traffic of lymphocytes to the tumour, and (d) induction of a potent antiangiogenic effect.

In an orthotopic HCC model in Buffalo rats, we have shown that intratumoral administration of recombinant adenovirus encoding IL-12 (AdIL-12) caused complete tumour eradication in most animals and increased long term survival. ${ }^{70}$ Interestingly, when two tumours were implanted in the same liver, treatment of only one resulted in regression of both. This effect has been attributed to the fact that a proportion of the adenovirus injected into a neoplastic nodule escapes to the general circulation and (because of the strong adenoviral hepatotropism) infects the whole liver. The IL-12 produced by the tumour and by hepatocytes surrounding the neoplastic nodules strongly activates natural killer cells, induces specific antitumoral immunity and enhanced expression of adhesion molecules in the tumoral vessels, and displays a powerful antiangiogenic effect with resulting tumour regression. ${ }^{70}$ AdIL- 12 given by the intrahepatic arterial route has also been shown to be efficient in the treatment of a very aggressive model of multifocal HCC in rats (DENA induced HCC) causing a significant reduction in tumour burden and prolongation of survival. ${ }^{70}$ AdIL- 12 was also found to induce potent antitumoral effects in animal models of metastatic colon cancer model. ${ }^{71}$

Although IL-12 is a potent antitumoral substance, its use is also liable to cause systemic toxic effects mainly derived from the ability of this cytokine to induce IFN- $\gamma$ production. In order to enhance the antitumoral activity of IL-12 while reducing the risk of toxicity, we have tested the therapeutic effect of a suboptimal dose of AdIL-12 in combination with an adenovirus expressing the chemokine IP-10 (AdIP-10). The rationale was to attract immunoeffector cells to the neoplasm through IP-10 and to activate the attracted lymphocytes with IL-12. We found that this combined therapy allows reduction of the dose of AdIL-12 without loss of antitumoral efficacy but with less risk of toxicity. ${ }^{72}$ As activation of dendritic cells, the most efficient antigen presenting cells, is critical for induction of antitumoral immunity, another possible way to enhance the antitumoral effect of low doses of IL-12 is to infect dendritic cells with AdIL-12 ex vivo and to inject these engineered dendritic cells into the tumour. ${ }^{73}$ In animal models of colon cancer this strategy has proved to be extremely potent at eliminating tumoral lesions and at eliciting antitumoral immune responses.

Stimulation of dendritic cells is widely dependent on activation by costimulatory molecules such as B7 and CD40 ligand (CD40L). ${ }^{74} 75$ We observed that adenovirus mediated gene transfer of CD40L (AdCD40L) completely abolished the tumorigenicity of ex vivo infected rat HCC cells and that intratumoral injection of AdCD40L into established intrahepatic HCC tumour nodules in rats resulted in tumour regression and prolongation of survival. Treatment of rat HCC with AdCD40L induced protective antitumour immunity and was devoid of significant toxicity. ${ }^{75}$

Genetic vaccination has also been reported to prevent tumour growth. In a murine model of subcutaneous HCC it has been shown that DNA vaccination with a cDNA encoding murine AFP induced tumour regression and prolonged animal survival. The antitumoral effect was mediated by cytotoxic $\mathrm{T}$ cells, and protective antitumoral immunity was shown to depend on both CD4+ and CD8 + T cells. $^{76}$

\section{"Genetic vaccination has also been reported to prevent tumour growth"}

Recently, improved immunisation protocols alternating DNA priming and recombinant vaccinia Ankara booster generated a manifold higher immune response than any of these strategies alone. ${ }^{77}$ However, these concepts have not yet been applied in the induction of antitumoral immunity for HCC treatment.

Some of these anticancer strategies, such as hepatic arterial administration of adenovirus with wild-type p53 ${ }^{78}$ or intratumoral injection of adenoviral vectors encoding suicide gene HSV/tk or IL-12 have been applied, or are at present been tested, in HCC patients in phase I/II clinical trials.

\section{OUTLOOK}

Gene therapy has emerged as a powerful and very plastic tool to regulate biological functions in diseased tissues with application in practically all medical fields. An increasing number of experimental and clinical studies clearly underline the important role of genes to serve as a curative drug in the future. However, intense research is needed to evaluate the potential of gene therapy, to improve efficacy, and to minimise the toxicity of the procedure.

\section{"Genes can be transferred to tissues and the transferred gene is functional in vivo"}

Future efforts should be directed towards: (1) development of vectors with high transduction efficiency, high transgene capacity, and acceptable toxicity profile, (2) development of systems allowing desired duration and regulation of gene expression, (3) identification of the ideal therapeutic gene or gene combinations for each therapeutic indication, (4) testing of different routes for vector administration, and (5) development of innovative methods for large scale industrial production to allow accessible costs for wide access to these new drugs. Intensive testing in animal models to ensure drug safety and functionality is needed before contemplating clinical applications. On the other hand, carefully designed clinical trials, under strict regulatory conditions, can provide very useful information on the potential of gene therapy in humans, especially in devastating diseases lacking effective therapy, as is the case for non-resectable chemoresistant tumours. New imaging techniques such as positron emission tomography have the potential of detecting and quantitating the function of the transferred gene to the tissues in vivo, thus providing a powerful tool to guide the progress of clinical gene therapy. In summary, genes can be transferred to tissues and the transferred gene is functional in vivo; this opens a new field of medical applications which is just beginning.

\section{ACKNOWLEDGEMENTS}

This work was performed with the help of grants from: MJ Huarte, J Vidal, M Mendez, Arburua Aspiunza, Fundacion Echebano, Fundacion Areces, and CICYT grants (SAF98-0146, SAF99-0039, and SAF99-0084). VS was partly supported by VERUM Foundation, Munich, Germany. 


\section{Authors' affiliations}

V Schmitz, Gene Therapy Unit, Department of Internal Medicine, Clinica Universitaria, Faculty of Medicine, Universidad de Navarra, Pamplona Spain and University Hospital Bonn, Internal Medicine, Germany C Qian, J Ruiz, B Sangro, I Melero, G Mazzolini, I Narvaiza, J Prieto, Gene Therapy Unit, Department of Internal Medicine, Clinica Universitaria, Faculty of Medicine, Universidad de Navarra, Pamplona Spain

\section{REFERENCES}

1 Miller AD. Human gene therapy comes of age. Nature 1992;357:455-60

2 Mulligan RC. The basic science of gene therapy. Science 1993;260:926-32

3 Herskowitz I. Functional inactivation of genes by dominant negative mutations. Nature 1987:329:219-22.

4 Haseloff J, Gerlach WL. Simple RNA enzymes with new and highly specific endoribonuclease activities. Nature 1988;334:585-91.

5 Symons RH. Ribozymes. Curr Opin Struct Biol 1994;4:322-30.

6 Crooke ST. Basic principles of antisense therapeutics. In: Crooke ST, eds. Antisense research and application. Handbook of experimental pharmacology, Vol 131. Berlin: Springer, 1998:1-50.

7 Eguchi Y. Antisense RNA. Annu Rev Biochem 1991;60:631-52.

8 Sullenger BA, Gallardo FH, Ungers GE, et al. Analysis of trans-acting response decoy RNA-mediated inhibition of human immunodeficiency virus type 1 transactivation. J Virol 1991;65:6811-16.

9 Prince HM. Gene transfer: A review of methods and applications. Pathology 1998;30:335-47

10 Crespo J, Blaya C, Crespo A, et al. Long-term expression of the alphal-antitrypsin gene in mice employing anionic and cationic liposome vectors. Biochem Pharmacol 1996;51:1309-14.

11 Holmberg EG, Rever QR, Geisert EE, et al. Delivery of plasmid DNA to glial cells using $\mathrm{pH}$-sensitive immunoliposomes. Biochem Biophys Res Comm 1994;201:888-93.

12 Saeki $Y$, Matsumoto N, Nakano Y, et al. Development and characterization of cationic liposomes conjugated with HVJ (Senda virus): reciprocal effect of cationic lipid for in vitro and in vivo gene transfer. Human Gene Ther 1997;8:2133-41

13 Tang DC, Devit $M$, Johnston SA. Genetic immunization is a simple method for eliciting an immune response. Nature 1992;356:152-4.

14 Yoshida Y, Kobayashi E, Endo H, et al. Introduction of DNA into rat liver with a hand-held gene gun: distribution of the expressed enzyme [32P]DNA, and C2+ flux. Biochem Biophys Res Comm 1997;234:695-700

15 Wu GY, Wu CH. Receptor mediated gene delivery and expression in vivo. J Biol Chem 1988;263:14621-4.

16 Wolff JA, Malone RW, Williams $\mathrm{P}$, et al. Direct gene transfer into mouse muscle in vivo. Science 1990;247:1465-8

17 Hickman MA, Malone RW, Lehmann-Bruinsma K, et al. Gene expression following direct injection of DNA into liver. Hum Gene Ther 1994:4:1477-83.

18 Gordon EM, Anderson WF. Gene therapy using retroviral vectors. Curr Opin Biotechnol 1995;6:611-16.

19 Kalpana GV. Retroviral vectors for liver-directed gene therapy. Semin Liver Dis 1999;19:27-37

20 Grossman M, Rader DJ, Muller DW, et al. A pilot study of ex vivo gene therapy for homozygous familiar hypercholesterolaemia. Nat Genet 1994;6:335-41.

21 Kafri T, Blomer U, Peterson DA, et al. Sustained expression of genes delivered directly into liver and muscle by lentiviral vectors. Nat Genet 1997; 17:314-17.

22 Topf N, Worgall S, Hackett NR, et al. Regional prodrug gene therapy: intravenous administration of an adenoviral vector expressing the $\mathrm{E}$. coli cytosine deaminase gene and systemic administration of 5-fluorocytosine suppresses growth of hepatic metastasis of colon carcinoma. Gene Ther 1998;5:507-13

23 Zhang WW. Development and application of adenoviral vectors for gene therapy of cancer. Cancer Gene Ther 1999;6:113-18.

24 Ponnazhagan S, Mukheriee P, Yoder MC et al. Adeno-associated virus 2-mediated gene transfer in vivo: organ-tropism and expression of transduced sequences in mice. Gene 1997;190:203-10

25 Yang Y, Nunes FA, Berencsi $K$, et al. Cellular immunity to viral antigens limits E1-deleted adenoviruses for gene therapy. Proc Natl Acad Sci USA 1994:91:4407-1 1.

26 Kaufman R. Advances toward gene therapy of hemophilia at the millenium. Hum Gene Ther 1999;10:2091-107.

27 Kochanek S. High-capacity adenoviral vectors for gene transfer and somatic gene therapy. Hum Gene Ther 1999; 10:2451-9.

28 Steinwaerder DS, Carlson CA, Otto DL, et al. Tumor-specific gene expression in hepatic metastases by a replication-activated adenovirus vector. Nat Med 2001; 7:240-3

29 Alemany R, Lai S, Lou YC, et al. Complementary adenoviral vectors for oncolysis. Cancer Gene Ther 1999;6:21-5.

30 Patiin GA, Kay MA. Hepatic gene therapy using adeno-associated virus vectors. Semin Liver Dis 1999;19:61-9.
31 Snyder RO, Miao $\mathrm{CH}$, Patijn GA, et al. Persistent and therapeutic concentrations of human factor IX in mice after hepatic gene transfer of recombinant AAV vectors. Nat Genet 1997;16:270-6

32 Alexander IE, Russell DW, Spence AM, et al. Effects of gamma irradiation on the transduction of dividing and nondividing cells in brain and muscle of rats by adeno-associated virus vectors. Hum Gene Ther 1996:7:841-50.

33 Koeberl DD, Alexander IE, Halbert CL, et al. Persistent expression of human clotting factor IX from mouse liver after intravenous injection of adeno-associated virus vectors. Proc Natl Acad Sci USA 1997; 94:1426-31.

34 Peng D, Qian C, Sun Y, et al. Transduction of hepatocellular carcinoma $(\mathrm{HCC})$ using recombinant adeno-associated virus ( $\mathrm{rAAV})$ : in vitro and in vivo effects of genotoxic agents. J Hepatol 2000;32:975-85

35 Miyanohara A, Johnson PA, Elam RL, et al. Direct gene transfer to the liver with herpes simplex virus type 1 vectors: transient production of physiologically relevant levels of circulating factor IX. New Biol 1992:4:238-46.

36 Malnick SD, Beergabel M, Lurie $Y$. Treatment of chronic hepatitis $C$ virus infection. Ann Pharmacother 2000;34:1 156-64

37 Glue P, Rouzier-Panis R, Raffanel C, et al. A dose-ranging study of pegylated interferon alfa- $2 b$ and ribavirin in chronic hepatitis $C$. The Hepatitis C Intervention Therapy Group. Hepatology 2000;32:647-53.

38 Aurisicchio L, Delmastro P, Salucci V, et al. Liver-specific alpha 2 interferon gene expression results in protection from induced hepatitis. $J$ Virol 2000;74:4816-23

39 Weizsacker FV, Wieland S, Lock J, et al. Gene therapy for chronic viral hepatitis: ribozymes, antisense oligonucleotides, and dominant negative mutants. Hepatology 1997; 26:251-5.

40 Alt $M$, Eisenhardt S, Serwe $M$, et al. Comparative inhibitory potential of differently modified antisense oligodeoxynucleotides on hepatitis $C$ virus translation. Eur J Clin Invest 1999;29:868-76

41 Vidalin O, Fournillier A, Renard N, et al. Use of conventional or replicating nucleic acid-based vaccines and recombinant Semliki forest virus-derived particles for the induction of immune responses against hepatitis C virus core and E2 antigens. Virology 2000;276:259-70.

42 Geissler M, Schirmbeck R, Reinmann J, et al. Cytokine and hepatitis B virus DNA co-immunizations enhance cellular and humoral immune responses to the middle but not the large hepatitis $B$ virus surface antigen in mice. Hepatology 1998;28:202-10.

43 Chow YH, Chiang BL, Lee YL, et al. Development of Th 1 and Th2 populations and the nature of immune responses to hepatitis $B$ virus DNA vaccines can be modulated by codelivery of various cytokine genes. $J$ Immunol 1998;160:1320-9.

44 Bruña-Romero O, Lasarte JJ, Wilkinson G, et al. Induction of cytotoxic T-cell response against hepatitis $c$ virus structural antigens using a defective recombinant adenovirus. Hepatology 1997:25:470-7.

45 Lasarte JJ, Corrales FJ, Casares N, et al. Different doses of adenoviral vector expressing IL-12 enhance or depress the immune response to a coadministered antigen: the role of nitric oxide. J Immunol $1999 ; 162: 5270-7$

46 García R, Berraondo P, Blanco B, et al. Protection against woodchuck hepatitis virus (WHV) infection by combined genetic immunization with WHV core and IL-12. J Virol 2001;75:9068-76.

47 Okuda K. Hepatocellular carcinoma. J Hepatol 2000;32(1 suppl): 225-37

48 Lau WY. Primary liver tumors. Semin Surg Oncol 2000;19:135-44.

49 Choti MA, Bulkley GB. Management of metastatic disease. In: Schiff ER Sorrell MF, Maddrey WC, eds. Schiff's disease of the liver. Philadelphia: Lippincott-Raven, 1999.

50 McCarter MD, Fong Y. Metastatic liver tumors. Semin Surg Onco 2000;19:177-88.

51 Lai EC, Fan ST, Lo CM, et al. Hepatic resection for hepatocellular carcinoma. An audit of 343 patients. Ann Surg 1995:221:291-8.

52 Qian C, Drozdzik M, Caselmann WH, et al. The potential of gene therapy in the treatment of hepatocellular carcinoma. J Hepatol 2000;32:344-51

53 Ruiz J, Qian C, Drozdzik M, et al. Gene therapy of viral hepatitis and hepatocellular carcinoma. J Viral Hepat 1999;6:17-34.

54 Heidemann DAM, Gerritsen WR, Craanen ME. Gene therapy for gastrointestinal tract cancer: a review. Scand J Gastroenterol 2000;232(suppl):93-100

55 Moolten FL. Drug sensitivity ("suicide") genes for selective cancer chemotherapy. Cancer Gene Ther 1994;1:279-87.

56 Qian C, Bilbao R, Bruña $O$, et al. Induction of sensitivity to ganciclovir in human hepatocellular carcinoma cells by adenovirus-mediated gene transfer of herpes simplex virus thymidine kinase. Hepatology 1995;22:118-23.

57 Bi WL, Parysek LM, Warnick $R$, et al. In vivo evidence that metabolic cooperation is responsible for the bystander effect observed with HSV tk retroviral gene therapy. Hum Gene Ther 1993:4:725-31.

58 Drozdzik M, Qian C, Xie X, et al. Combined gene therapy with suicide gene and interleukin-12 is more efficient than therapy with one gene alone in a murine model of hepatocellular carcinoma. J Hepato 2000;32:279-86.

59 Kuriyama S, Nakatani T, Masui K, et al. Evaluation of prodrugs ability to induce effective ablation of cells transduced with viral thymidine kinase gene. Anticancer Res 1996;16:2623-8

60 Qian C, lodate M, Bilbao R, et al. Gene transfer and therapy with adenoviral vector in rats with diethylnitrosamine-induced hepatocellular carcinoma. Hum Gene Ther 1997;8:349-58.

61 Hayashi S, Emi N, Yokoyama I, et al. Effect of gene therapy with the herpes simplex virus-thymidine kinase gene on hepatic metastasis in murine colon cancer. Surg Today 1997;27:40-3. 
62 Hayashi S, Emi N, Yokoyama I, et al. Inhibition of establishment of hepatic metastasis in mice by combination gene therapy using both herpes simplex virus-thymidine kinase and granulocyte macrophage-colony stimulating factor genes in murine colon cancer Cancer Gene Ther 1997:4:339-44.

63 Brand K, Arnold W, Bartels T, et al. Liver-associated toxicity of the HSV-tk/GCV approach and adenoviral vectors. Cancer Gene Ther 1997;4:9-16.

64 Kaneko S, Hallenbeck P, Kotani T, et al. Adenovirus-mediated gene therapy of hepatocellular carcinoma using cancer-specific gene expression. Cancer Res 1995;55:5283-7.

65 Roth C, Rochlitz C, Kourilsky P. Immune response against tumors. Adv Immunol 1994:57:281-351.

66 Gabrilovich DI, Chen HL, Girgis KR, et al. Production of vascular endothelial growth factor by human tumors inhibits the functional maturation of dendritic cells. Nat Med 1996;2:1096-103.

67 Musiani P, Modesti A, Giovarella M, et al. Cytokine, tumor-cell death and immunogenicity: a question of choice. Immunol Today 1997; 18:32-6.

68 Schmidt-Wolf G, Schmidt-Wolf GH. Cytokines and gene therapy. Immunol Today 1995; 16:173-5.

69 Colombo $M$, Forni G. Cytokine gene transfer in tumor inhibition and tumor therapy: where are we now? Immunol Today 1994;15:48-51.

70 Barajas M, Mazzolini G, Genové G, et al. Gene therapy of orthotopic hepatocellular carcinoma in rats using adenovirus coding for interleukin-12 (IL-12). Hepatology 2001;33:52-61.
71 Brunda MJ, Luistro L, Warrier RR, et al. Antitumor and antimetastatic activity of interleukin 12 against murine tumors. J Exp Med 1993; 178: 1223-30

72 Narvaiza I, Mazzolini G, Barajas M, et al. Intratumoral coinjection of two adenoviruses, one encoding the chemokine IFN-gamma-inducible protein-10 and another encoding IL-12, results in marked antitumoral synergy. J Immunol 2000;164:3112-22.

73 Mazzolini G, Qian C, Narvaiza I, et al. Adenoviral gene transfer of interleukin 12 into tumors synergizes with adoptive T cell therapy both at the induction and effector level. Hum Gene Ther 2000;11:113-25.

74 Sun Y, Qian C, Peng D, et al. Gene transfer to liver cancer cells of B7-1 plus interleukin 12 changes immunoeffector mechanisms and suppresses helper T cell type 1 cytokine production induced by interleukin 12 alone. Hum Gene Ther 2000;11:127-38.

75 Schmitz V, Barajas M, Wang L, et al. Adenovirus mediated CD40 ligand gene therapy in a rat model of orthotopic hepatocellular carcinoma. Hepatology 2001;34:72-81.

76 Grimm CF, Ortmann D, Mohr L, et al. Mouse alpha-fetoprotein-specific DNA-based immunotherapy of heptocellular carcinoma leads to tumor regression in mice. Gastroenterology 2000;199:1 104-12.

77 Amara RR, Villinger F, Altman JD, et al. Control of a mucosal challenge and prevention of AIDS by a multiprotein DNA/MVA vaccine. Science 2001;292:69-74.

78 Habib NA, Hodgson HJ, Lemoine N, et al. A phase I/II study of hepatic artery infusion with wtp53-CMV-Ad in metastatic malignant liver tumours. Hum Gene Ther 1999;10:2019-34.



Sign up to receive the table of contents by email every month. You can select from three alerts: Table of Contents (full), TOC Awareness (notice only); Gut related announcements.

www.gutjnl.com 